\title{
Strongly almost disjoint familes, revisited
}

\author{
by
}

\begin{abstract}
A. Haj n a l (New Brunswick, NJ), I. J u hás z (Budapest) and S. Shelah (New Brunswick, NJ, and Jerusalem)
\end{abstract}

\begin{abstract}
The relations $M(\kappa, \lambda, \mu) \rightarrow B$ [resp. $B(\sigma)]$ meaning that if $\mathcal{A} \subset[\kappa]^{\lambda}$ with $|\mathcal{A}|=\kappa$ is $\mu$-almost disjoint then $\mathcal{A}$ has property $B$ [resp. has a $\sigma$-transversal] had been introduced and studied under GCH in $[\mathrm{EH}]$. Our two main results here say the following:

Assume GCH and let $\varrho$ be any regular cardinal with a supercompact [resp. 2-huge] cardinal above $\varrho$. Then there is a $\varrho$-closed forcing $P$ such that, in $V^{P}$, we have both GCH and $M\left(\varrho^{(+\varrho+1)}, \varrho^{+}, \varrho\right) \nrightarrow B$ [resp. $M\left(\varrho^{(+\varrho+1)}, \lambda, \varrho\right) \nrightarrow B\left(\varrho^{+}\right)$for all $\left.\lambda \leq \varrho^{(+\varrho+1)}\right]$.

These show that, consistently, the results of $[\mathrm{EH}]$ are sharp. The necessity of using large cardinals follows from the results of [Ko], [HJSh] and [BDJShSz].
\end{abstract}

1. Introduction. The aim of this paper is to show that, assuming the existence of certain large cardinals, the results of $[\mathrm{EH}]$ are sharp. Let us recall these results, and first their terminology.

If $\mu \leq \lambda \leq \kappa$ and $\sigma$ are infinite cardinals then $M(\kappa, \lambda, \mu) \rightarrow B(\sigma)$ [resp. $M(\kappa, \lambda, \mu) \rightarrow B$ ] abbreviates the following statement: Whenever $\mathcal{A} \subset$ $[\kappa]^{\lambda}$ with $|\mathcal{A}|=\kappa$ is $\mu$-almost disjoint (for short: $\mu$-a.d.) then $\mathcal{A}$ has a $\sigma$-transversal [resp. $\mathcal{A}$ has property $B$ ]. Here $\mathcal{A}$ is $\mu$-a.d. means that the intersection of any two members of $\mathcal{A}$ has size $<\mu$; a $\sigma$-transversal of $\mathcal{A}$ is a set $T$ such that $0<|A \cap T|<\sigma$ for every $A \in \mathcal{A}$; and $\mathcal{A}$ has property $B$ if there is a set $T$ with $\emptyset \neq A \cap T \neq A$ for all $A \in \mathcal{A}$.

One of the main results of [EH] (see also [W, Chapter 1]) is as follows:

1.1. TheOREM. (GCH) If $\varrho$ is any regular cardinal then for any $\lambda \leq \kappa \leq$ $\varrho^{(+\varrho)}$ we have

$$
M(\kappa, \lambda, \varrho) \rightarrow B\left(\varrho^{+}\right) .
$$

2000 Mathematics Subject Classification: Primary 03E05, 03E35; Secondary 03E50, $03 \mathrm{E} 55$

Key words and phrases: strongly almost disjoint family, property $B, \sigma$-transversal.

Research supported by NSF grant DMS-9704477 (A. Hajnal); NSF grant DMS-9704477 and OTKA grant 25745 (I. Juhász); NSF grant DMS-9704477 and the Israel Science Foundation founded by the Israel Academy of Sciences and Humanities, publication no. 697 (S. Shelah). 
The natural question whether the restriction $\kappa \leq \varrho^{(+\varrho)}$ is essential here had also been raised in $[\mathrm{EH}]$, especially because the following was also proved there.

1.2. TheOREM. (GCH) If $\varrho$ is regular then for any $\lambda \leq \kappa$ we have

$$
M(\kappa, \lambda, \varrho) \rightarrow B\left(\varrho^{++}\right) .
$$

So if also $\lambda>\varrho^{+}$then $M(\kappa, \lambda, \varrho) \rightarrow B$.

Concerning the above question it was much later shown in $[\mathrm{Ko}]$ and than in [HJSh] and [BDJShSz] that the restriction $\kappa \leq \varrho^{(+\varrho)}$ in 1.1 can be omitted if some weak $\square$-like principles hold in addition to $\mathrm{GCH}$, hence e.g. if $V=L$. On the other hand, it was also shown in [HJSh] that the existence of a supercompact cardinal implies the consistency of $M\left(\aleph_{\omega+1}, \aleph_{1}, \aleph_{0}\right) \nrightarrow B$, hence also of $M\left(\aleph_{\omega+1}, \aleph_{1}, \aleph_{0}\right) \nrightarrow B\left(\aleph_{1}\right)$, with GCH. The appearance of large cardinals here is of course essential because one has to negate the above mentioned $\square$-like principles.

Our first main result generalizes this negative result from $\varrho=\aleph_{0}$ to any regular cardinal $\varrho$. This was not immediate because the method of proof used in [HJSh] does not apply if $\varrho>\aleph_{0}$, so a new ingredient was needed. The general result can be formulated as follows.

1.3. Theorem. Assume that GCH holds, @ is any regular cardinal and $\kappa$ is a supercompact cardinal with $\varrho<\kappa$. Then there is a $\varrho$-closed notion of forcing $P$ such that, in $V^{P}$, we have $G C H$ and

$$
M\left(\varrho^{(+\varrho+1)}, \varrho^{+}, \varrho\right) \nrightarrow B .
$$

(Note that since $P$ is $\varrho$-closed, no cardinals or cofinalities will be changed in $V^{P}$ up to $\varrho$.)

Of course, trivially here again $M\left(\varrho^{(+\varrho+1)}, \varrho^{+}, \varrho\right) \nrightarrow B\left(\varrho^{+}\right)$holds, but the relations $M\left(\varrho^{(+\varrho+1)}, \lambda, \varrho\right) \nrightarrow B\left(\varrho^{+}\right)$are not excluded for $\varrho^{+}<\lambda \leq \varrho^{(+\varrho+1)}$. Our second main result, formulated below, takes care of these. (Compare this with 1.2!)

1.4. Theorem. Assume $G C H, \varrho$ is regular and $\kappa$ is 2 -huge with $\varrho<\kappa$. Then there is a @-closed notion of forcing $P$ such that, in $V^{P}$, we have $G C H$ and

$$
M\left(\varrho^{(+\varrho+1)}, \lambda, \varrho\right) \nrightarrow B\left(\varrho^{+}\right)
$$

for all $\lambda \leq \varrho^{(+\varrho+1)}$.

These results indeed show that, modulo some large cardinals, the results of $[\mathrm{EH}]$ are best possible. However, the question of exactly what large cardinals are needed, in particular whether the rather large step from the supercompact of 1.3 to the 2 -huge of 1.4 is necessary, remains open. 
2. The proof of $\mathbf{1 . 3}$. We start by recalling the following simple result from [HJSh]:

2.1. Lemma. Let $S \subset \kappa$ be a stationary set such that $\diamond(S)$ holds and $\left\{A_{\alpha}: \alpha \in S\right\}$ be a family of infinite sets with $A_{\alpha} \subset \alpha$ for each $\alpha \in S$. Then we can find sets $B_{\alpha} \subset A_{\alpha}$ with $\left|B_{\alpha}\right|=\left|A_{\alpha}\right|$ for all $\alpha \in S$ so that the family $\left\{B_{\alpha}: \alpha \in S\right\}$ does not have property $B$.

Now fix a regular cardinal $\varrho$, and to simplify notation denote $\varrho^{(+\varrho+1)}$ by $\widehat{\varrho}$. Also, given two regular cardinals $\lambda$ and $\kappa$ with $\lambda<\kappa$ we set

$$
S_{\lambda}^{\kappa}=\{\alpha \in \kappa: \operatorname{cf}(\alpha)=\lambda\} .
$$

Thus, by $2.1, M\left(\widehat{\varrho}, \varrho^{+}, \varrho\right) \nrightarrow B$ if we can find a stationary set $S \subset S_{\varrho^{+}}^{\widehat{\varrho}}$ satisfying $\diamond(S)$ and a $\varrho$-a.d. family $\left\{A_{\alpha}: \alpha \in S\right\}$ such that $A_{\alpha} \in[\alpha]^{\varrho^{+}}$for each $\alpha \in S$. Note that, as is well known (see e.g. [Gr]), GCH implies $\diamond(S)$ whenever $S \subset S_{\lambda}^{\kappa}$ is stationary if $\lambda>\omega$.

So far, everything has been done as in [HJSh] for the case $\varrho=\aleph_{0}$. It is the following theorem that allows us to get the result for an arbitrary regular cardinal $\varrho$.

2.2. TheOREM. Let $\mu$ be a singular cardinal of cofinality $\varrho$ and such that $\mu=\mu^{<\varrho}$. Then there is a partial order $Q=Q(\mu)$ with properties (i)-(v) below:

(i) $Q$ is $\varrho$-closed;

(ii) $Q$ is $\mu^{+}-C C$;

(iii) $|Q| \leq 2^{\mu}$;

(iv) in $V^{Q}, \mu$ is collapsed to $\varrho$, and $\varrho^{+}=\mu^{+}$;

(v) there is, in $V^{Q}$, a set $X \in\left[\varrho^{+}\right]^{\varrho^{+}}$such that for any set $H \in \mathcal{P}\left(\mu^{+}\right) \cap$ $V$ we have $|H \cap X|^{V^{Q}}<\varrho$ if and only if $|H|^{V}<\mu$.

Pr o o f. Put $Q=Q_{1} \times Q_{2}$, where $Q_{1}$ is the natural $\varrho$-closed partial order that forces a map of $\varrho$ onto $\mu$, i.e. $q \in Q_{1}$ iff $q$ maps some $\alpha \in \varrho$ into $\mu$, and extension is the partial ordering. Moreover,

$$
Q_{2}=\left[\mu^{+}\right]^{<\varrho} \times\left[\mu^{+}\right]^{<\mu}
$$

with the following ordering: for $\langle a, A\rangle,\left\langle a^{\prime}, A^{\prime}\right\rangle \in Q_{2}$ we have $\langle a, A\rangle \leq\left\langle a^{\prime}, A^{\prime}\right\rangle$ iff $a \supset a^{\prime}, A \supset A^{\prime}$ and $A^{\prime} \cap\left(a \backslash a^{\prime}\right)=\emptyset$.

Clearly, both $Q_{1}$ and $Q_{2}$ are $\varrho$-closed, hence so is $Q$, i.e. (i) holds.

To show (ii), first note that from $\mu=\mu^{<\varrho}$ we have $\left|Q_{1}\right|=\mu$ and so it suffices to prove that $Q_{2}$ is $\mu^{+}$-CC. Thus let $\left\{\left\langle a_{i}, A_{i}\right\rangle: i \in \mu^{+}\right\} \subset Q_{2}$; clearly we may assume that $\left|a_{i} \cup A_{i}\right|<\lambda$ for a fixed regular cardinal $\lambda<\mu$ for all $i \in \mu^{+}$. Now, for every $\gamma \in S_{\lambda}^{\mu^{+}}$the set $B_{\gamma}=\left(a_{\gamma} \cup A_{\gamma}\right) \cap \gamma$ is bounded in $\gamma$, i.e. there is an $f(\gamma)<\gamma$ with $B_{\gamma} \subset f(\gamma)$. So by Fodor's theorem there 
is a stationary set $S \subset S_{\lambda}^{\mu^{+}}$on which $f$ takes the constant value $\alpha$. Using $\mu^{<\varrho}=\mu$ we may also assume that $a_{\gamma} \cap \gamma=a_{\gamma} \cap \alpha=c$ for all $\gamma \in S$.

Now pick $\gamma, \delta \in S$ such that both $\gamma<\delta$ and $a_{\gamma} \cup A_{\gamma} \subset \delta$ (this is possible because each $a_{\gamma} \cup A_{\gamma}$ is bounded in $\mu^{+}$), and set $a=a_{\gamma} \cup a_{\delta}, A=A_{\gamma} \cup A_{\delta}$. Clearly, $\langle a, A\rangle \in Q_{2}$. We next show that $\langle a, A\rangle$ extends both $\left\langle a_{\gamma}, A_{\gamma}\right\rangle$ and $\left\langle a_{\delta}, A_{\delta}\right\rangle$. Indeed, this follows because $a \backslash a_{\gamma}=a_{\delta} \backslash c \subset \mu^{+} \backslash \delta$ and $A_{\gamma} \subset \delta$ imply $A_{\gamma} \cap\left(a \backslash a_{\gamma}\right)=\emptyset$, moreover $a \backslash a_{\delta}=a_{\gamma} \backslash c \subset \delta \backslash \gamma$ and $A_{\delta} \subset \alpha \cup\left(\mu^{+} \backslash \delta\right)$ imply $A_{\delta} \cap\left(a \backslash a_{\delta}\right)=\emptyset$. $=2^{\mu}$.

(iii) follows easily because $\left|Q_{1}\right|=\mu$ and $\left|Q_{2}\right|=\left(\mu^{+}\right)^{<\varrho}\left(\mu^{+}\right)^{<\mu} \leq\left(\mu^{+}\right)^{\mu}$

(iv) is again trivial because $Q_{1}$ collapses $\mu$ to $\varrho$ and by (ii), $\mu^{+}$is preserved.

Finally, to see (v), let $G=G_{1} \times G_{2}$ be $Q$-generic over $V$ and set, in $V[G]$,

$$
X=\bigcup\left\{a:(\exists A)\left(\langle a, A\rangle \in G_{2}\right)\right\} .
$$

Clearly, for every $\alpha \in \mu^{+}$the set

$$
D_{\alpha}=\left\{\langle a, A\rangle \in Q_{2}: a \backslash \alpha \neq \emptyset\right\}
$$

is dense in $Q_{2}$ and so $X$ is unbounded in $\mu^{+}=\varrho^{+}$, i.e. $X \in\left[\varrho^{+}\right] \varrho^{+}$.

Now, if $H \in\left[\mu^{+}\right]^{<\mu} \cap V$ then again

$$
D_{H}=\left\{\langle a, A\rangle \in Q_{2}: H \subset A\right\}
$$

is dense in $Q_{2}$ because $\langle a, A \cup H\rangle \leq\langle a, A\rangle$ for each $\langle a, A\rangle \in Q_{2}$. But then $G_{2} \cap D_{H} \neq \emptyset$, and if $\langle a, A\rangle \in G_{2} \cap D_{H}$ then clearly $X \cap H \subset X \cap A \subset a$, hence $|X \cap H|<\varrho$.

If, on the other hand, $H \subset \mu^{+}, H \in V$ and $|H| \geq \mu$ then clearly

$$
E_{H}=\left\{\langle a, A\rangle \in Q_{2}: a \cap H \neq \emptyset\right\}
$$

is dense in $Q_{2}$. Now, if we had $|X \cap H|<\varrho$ then by (i) we would also have $X \cap H \in V$ and so $H \backslash X \in V$ and $|H \backslash X| \geq \mu$. This, however, contradicts the denseness of $E_{H \backslash X}$.

The following corollary is now immediate.

2.3. Corollary. With the assumptions of 2.2 , we can, in $V^{Q}$, associate with every ground model set $A \in V$ with $|A|=\varrho^{+}=\mu^{+}$a subset $A^{*} \in[A]^{\varrho^{+}}$ such that for any set $B \in V$ we have $\left|A^{*} \cap B\right|<\varrho$ iff $|A \cap B|^{V}<\mu$. In particular, if $\mathcal{A}$ is a $\mu$-a.d. family of sets of size $\mu^{+}$in $V$ then $\mathcal{A}^{*}=\left\{A^{*}\right.$ : $A \in \mathcal{A}\}$ is a $\varrho$-a.d. family of sets of size $\varrho^{+}$in $V^{Q}$.

Proof. Let $h: \mu^{+} \rightarrow A$ be a bijection of $\mu^{+}$onto $A$ in $V$. Clearly,

$$
A^{*}=\{h(\xi): \xi \in X\}
$$

is as required by $(\mathrm{v})$ of 2.2 . 
Let us now return to the proof of 1.3. Put $\lambda=\kappa^{(+\varrho)}$. Since $\kappa$ is $\lambda^{+}$supercompact we can fix a normal, $\kappa$-complete ultrafilter $\mathcal{U}$ on $\left[\lambda^{+}\right]^{<\kappa}$. Using GCH we get $\left(\lambda^{+}\right)^{\varrho}=\lambda^{+}$, hence we may also fix a bijection $G$ of $\left[\lambda^{+}\right]^{\varrho}$ onto $\lambda^{+}$. Standard reflection arguments and Solovay's Theorem 2 from [S] then imply the existence of a set $A \in \mathcal{U}$ such that

(i) the map $P \mapsto \bigcup P$ is one-one on $A$;

(ii) each $P \in A$ is $G$-closed;

(iii) $P \cap \kappa$ is an inaccessible cardinal and

$$
\operatorname{tp}(P)=(P \cap \kappa)^{(+\varrho+1)} \quad \text { for each } P \in A .
$$

Now the set $S_{1}=\{\bigcup P: P \in A\}$ is clearly stationary in $\lambda^{+}$since $\mathcal{U}$ is normal and, by (i), we have $A=\left\{P_{\alpha}: \alpha \in S_{1}\right\}$ where $\bigcup P_{\alpha}=\alpha$ for $\alpha \in S_{1}$.

Now consider the map $\alpha \mapsto P_{\alpha} \cap \kappa$ on $S_{1}$. By (iii) we have a fixed inaccessible cardinal $\tau$ such that

$$
S=\left\{\alpha \in S_{1}: P_{\alpha} \cap \kappa=\tau\right\}
$$

is also stationary. We claim that the family $\left\{P_{\alpha}: \alpha \in S\right\} \subset\left[\lambda^{+}\right]^{\tau^{(+\varrho+1)}}$ is also $\tau^{(+\varrho)}$-a.d. Indeed, if $\left|P_{\alpha} \cap P_{\beta}\right| \geq \tau^{(+\varrho)}$ held for some distinct $\alpha, \beta \in S$ then by (ii) we would also have $\left|P_{\alpha} \cap P_{\beta}\right|=\tau^{(\varrho+1)}$, using the fact that $P_{\alpha} \cap P_{\beta}$ is $G$-closed. This, however, contradicts $\operatorname{tp}\left(P_{\alpha}\right)=\operatorname{tp}\left(P_{\beta}\right)=\tau^{(\varrho+1)}$ and $\bigcup P_{\alpha}=\alpha \neq \bigcup P_{\beta}=\beta$.

Note that the singular cardinal $\mu=\tau^{(+\varrho)}$ satisfies the conditions of 2.2 , hence in $V^{Q(\mu)}$ the GCH holds, $S$ remains stationary, and the family $\left\{P_{\alpha}^{*}: \alpha \in S\right\} \subset\left[\lambda^{+}\right] \varrho^{+}$is $\varrho$-a.d., according to 2.3. All that remains to be done is now to do a further $\varrho$-closed forcing that turns $\lambda^{+}$into $\varrho$ and preserves both GCH and the stationarity of $S$. This job will clearly be done by e.g. $\operatorname{Lv}\left(\kappa, \varrho^{++}\right)$, i.e. the Levy collapse of $\kappa$ to $\varrho^{++}$in $V^{Q(\mu)}$. Then $P=Q(\mu) *$ $\operatorname{Lv}\left(\kappa, \varrho^{++}\right)$is a $\varrho$-closed forcing such that $V^{P}$ satisfies GCH; moreover, in $V^{P},\left\{P_{\alpha}^{*}: \alpha \in S\right\} \subset[\varrho]^{\varrho^{+}}$is $\varrho$-a.d. But here $S \subset S_{\varrho^{+}}^{\widehat{\varrho}}$ is stationary and so by GCH we also have $\diamond(S)$, so Lemma 2.1 applies and hence $M\left(\widehat{\varrho}, \varrho^{+}, \varrho\right) \nrightarrow B$ in $V^{P}$.

3. A "stick"-like principle. The aim of this section is to introduce a "stick"-like combinatorial principle that will play an essential role in the proof of Theorem 1.4. We also look at some other results of purely combinatorial nature and thus separate the combinatorial arguments from the rest, to be given in the next section.

3.1. Definition. If $\kappa>\lambda \geq \mu \geq \omega$ then we denote by $\uparrow(\kappa, \lambda, \mu)$ the following statement: There is a $\mu$-a.d. family $\mathcal{A} \subset[\kappa]^{\lambda}$ with $|\mathcal{A}|=\kappa$ such that for every set $X \in[\kappa]^{\kappa}$ there is some $A \in \mathcal{A}$ with $A \subset X$; if $\mathcal{A}$ is like this then we say that $\mathcal{A}$ is a $\uparrow(\kappa, \lambda, \mu)$-family. 
The relevance of this to our subject, in particular to 1.4, becomes clear from the following result.

3.2. Lemma. $\uparrow(\kappa, \lambda, \mu)$ implies that $M(\kappa, \kappa, \mu) \nrightarrow B(\lambda)$.

Proof. Let $\mathcal{A}$ be a $\uparrow(\kappa, \lambda, \mu)$-family and fix a partition $\left\{X_{\xi}: \xi \in \kappa\right\} \subset$ $[\kappa]^{\kappa}$ of $\kappa$ into $\kappa$-many sets of size $\kappa$. Then we set

$$
\mathcal{B}=\left\{A \in \mathcal{A}:(\forall \xi \in \kappa)\left(\left|A \cap X_{\xi}\right| \leq 1\right)\right\} .
$$

Clearly $|\mathcal{B}|=|\mathcal{A}|=\kappa$, hence we may also fix a one-one enumeration $\mathcal{B}=$ $\left\{B_{\xi}: \xi \in \kappa\right\}$. Now, for every $\xi \in \kappa$ we set

$$
Y_{\xi}=X_{\xi} \cup B_{\xi}
$$

Then it is obvious that the family $\mathcal{Y}=\left\{Y_{\xi}: \xi \in \kappa\right\} \subset[\kappa]^{\kappa}$ is $\mu$-a.d., hence we shall be done if we can show that $\mathcal{Y}$ has no $\lambda$-transversal.

So assume that $T$ is such that $T \cap Y_{\xi} \neq \emptyset$ for all $\xi \in \kappa$. We claim that then the set $a=\left\{\xi \in \kappa: T \cap X_{\xi} \neq \emptyset\right\}$ has size $\kappa$.

Assume, indirectly, that $|a|<\kappa$. It is clear that for any set $H \in[\kappa]^{\kappa}$ which satisfies $\left|H \cap X_{\xi}\right| \leq 1$ for all $\xi \in \kappa$, we have

$$
\left|\left\{B_{\xi} \in \mathcal{B}: B_{\xi} \subset H\right\}\right|=\kappa \text {. }
$$

In particular, if $\alpha_{\xi}$ is the minimal member of $X_{\xi}$ for any $\xi \in \kappa$, then we may apply the above observation to the set

$$
H=\left\{\alpha_{\xi}: \xi \in \kappa \backslash a\right\} \in[\kappa]^{\kappa} .
$$

So there is some $\xi \in \kappa \backslash a$ such that $B_{\xi} \subset H$. But then, by the definition of $a$, we have both $T \cap H=\emptyset$, hence $T \cap B_{\xi}=\emptyset$, and $T \cap X_{\xi}=\emptyset$, i.e. $T \cap Y_{\xi}=\emptyset$, a contradiction.

Now, for every $\xi \in a$ pick an element $\beta_{\xi} \in T \cap X_{\xi}$ and set $K=\left\{\beta_{\xi}\right.$ : $\xi \in a\}$. We may then apply the above observation to the set $K \in[\kappa]^{\kappa}$ and find $B_{\xi} \in \mathcal{B}$ with $B_{\xi} \subset K$. So we conclude that $T \cap Y_{\xi} \supset B_{\xi}$, hence $\left|T \cap Y_{\xi}\right| \geq\left|B_{\xi}\right|=\lambda$, i.e. $T$ is not a $\lambda$-transversal.

REMARK. We have actually shown that $\mathcal{Y}$ has the following stronger property: For any set $T$, if $\left|\left\{\xi \in \kappa: T \cap Y_{\xi}=\emptyset\right\}\right|<\kappa$ then there is some $Y_{\xi} \in \mathcal{Y}$ with $\left|T \cap Y_{\xi}\right| \geq \lambda$.

Our next result yields a method for "stepping down" in the second parameter $\lambda$ of a negative relation of the form $M(\kappa, \lambda, \mu) \nrightarrow B(\sigma)$.

3.3. Lemma. Assume that $\tau<\lambda$ and we have both

$$
M(\kappa, \lambda, \mu) \nrightarrow B(\sigma)
$$

and

$$
M(\kappa, \lambda, \mu) \rightarrow B\left(\tau^{+}\right) .
$$

Then we also have $M(\kappa, \tau, \mu) \nrightarrow B(\sigma)$. 
Proof. Let $\mathcal{Y}=\left\{Y_{\xi}: \xi \in \kappa\right\} \subset[\kappa]^{\lambda}$ be a $\mu$-a.d. family with no $\sigma$ transversal. By transfinite recursion on $\alpha \in \tau$ we define sets $T_{\alpha}$ that are all $\tau^{+}$-transversals of $\mathcal{Y}$ as follows.

Let $T_{0}$ be any $\tau^{+}$-transversal of $\mathcal{Y}$; it exists by $(* *)$. If $T_{\beta}$ has been defined for each $\beta \in \alpha \in \tau$ then for every $Y_{\xi} \in \mathcal{Y}$ we have $\left|Y_{\xi} \backslash \bigcup\left\{T_{\beta}: \beta \in \alpha\right\}\right|=\lambda$ because, by the inductive hypothesis, $\left|Y_{\xi} \cap T_{\beta}\right| \leq \tau$ for each $\beta \in \alpha$. So we may now apply $(* *)$ to the family $\mathcal{Y}_{\alpha}=\left\{Y_{\xi} \backslash \bigcup\left\{T_{\beta}: \beta \in \alpha\right\}: \xi \in \kappa\right\}$ and obtain a $\tau^{+}$-transversal $T_{\alpha}$ of $\mathcal{Y}_{\alpha}$. Note that we may assume $T_{\alpha} \subset \cup \mathcal{Y}_{\alpha}$ and hence $T_{\alpha}$ is a $\tau^{+}$-transversal of $\mathcal{Y}$ as well.

Having completed the recursion, set $T=\bigcup\left\{T_{\alpha}: \alpha \in \tau\right\}$ and $Z_{\xi}=Y_{\xi} \cap T$ for each $\xi \in \kappa$. It is clear from the construction that $\left|Z_{\xi}\right|=\tau$, hence $\mathcal{Z}=$ $\left\{Z_{\xi}: \xi \in \kappa\right\}$ is a $\mu$-a.d. subfamily of $[\kappa]^{\tau}$, so we will be done if we can show that $\mathcal{Z}$ has no $\sigma$-transversal.

Since $\bigcup \mathcal{Z} \subset T$, it suffices to show that if $U \subset T$ intersects every member of $\mathcal{Z}$ then $\left|U \cap Z_{\xi}\right| \geq \sigma$ for some $Z_{\xi} \in \mathcal{Z}$. However, we know that there is a $\xi \in \kappa$ with $\left|U \cap Y_{\xi}\right| \geq \sigma$, which by $U \subset T$ and $Z_{\xi}=T \cap Y_{\xi}$ implies $\left|U \cap Z_{\xi}\right| \geq \sigma$, completing the proof.

Putting 1.2 and 3.3 together we immediately obtain the following result.

3.4. Corollary. (GCH) If $M(\kappa, \kappa, \varrho) \nrightarrow B\left(\varrho^{+}\right)$then for any $\lambda$ with $\varrho^{+}<\lambda<\kappa$ we have $M(\kappa, \lambda, \varrho) \nrightarrow B\left(\varrho^{+}\right)$as well.

This implies that to prove 1.4 it suffices to concentrate on $M(\widehat{\varrho}, \widehat{\varrho}, \varrho) \nrightarrow$ $B\left(\varrho^{+}\right)$, and so, by 3.2 , on $\uparrow\left(\widehat{\varrho}, \varrho^{+}, \varrho\right)$.

Let us now make a few observations about the principles $\uparrow(\kappa, \lambda, \mu)$ that are less closely related to the main subject matter of this paper.

If $\uparrow(\kappa, \lambda, \mu)$ is valid then we obviously have a $\uparrow(\kappa, \lambda, \mu)$ family $\mathcal{A}$ such that $\operatorname{tp} A=\lambda$ for every $A \in \mathcal{A}$. Now put

$$
S_{\mathcal{A}}=\{\bigcup A: A \in \mathcal{A}\},
$$

so $S_{\mathcal{A}} \subset S_{\varrho}^{\kappa}$, where $\varrho=\operatorname{cf}(\lambda) \leq \lambda<\kappa$. We claim that if $\kappa$ is regular then $S_{\mathcal{A}}$ is also stationary. Indeed, if $C \subset \kappa$ is c.u.b. then, as $|C|=\kappa$, there is some $A \in \mathcal{A}$ with $A \subset C$ and thus $\bigcup A \in S_{\mathcal{A}} \cap C \neq \emptyset$. So, if GCH holds and $\varrho>\omega$ then we also have $\diamond\left(S_{\mathcal{A}}\right)$, and consequently from 2.1 and 1.2 we easily obtain the following result.

3.5. Proposition. (GCH) If $\kappa$ is regular then $\boldsymbol{\bullet}(\kappa, \lambda, \mu)$ implies $M(\kappa, \lambda, \mu) \nrightarrow B$. Hence if $\kappa>\lambda>\varrho^{+}$where $\kappa$ and $\varrho$ are regular then $\bullet(\kappa, \lambda, \varrho)$ is false.

Thus, under GCH, for regular $\kappa$ and $\varrho$ the best we may hope for is $\bullet\left(\kappa, \varrho^{+}, \varrho\right)$; moreover, in view of $1.1, \widehat{\varrho}$ is the smallest possible value for $\kappa$ where this may happen. Moreover, by the next result, $\boldsymbol{\uparrow}\left(\kappa, \varrho^{+}, \varrho\right)$ fails for "most" regular $\kappa>\varrho^{+}$even in ZFC. 
3.6. Proposition. If $\kappa$ is regular and for every $\lambda<\kappa$ we have $\lambda^{\varrho}<\kappa$ then $\bullet\left(\kappa, \varrho^{+}, \varrho\right)$ is false.

Proof. Assume that $\mathcal{A} \subset[\kappa] \varrho^{+}$is $\varrho$-a.d. with $\operatorname{tp} A=\varrho^{+}$for all $A \in \mathcal{A}$. According to what we have seen above, it suffices to show that $S_{\mathcal{A}}$ is nonstationary in $\kappa$.

Assume, indirectly, that $S_{\mathcal{A}}$ is stationary and for each $\alpha \in S_{\mathcal{A}}$ let $A_{\alpha} \in \mathcal{A}$ be such that $\bigcup A_{\alpha}=\alpha$. For every $\alpha \in S_{\mathcal{A}}$ let $f(\alpha)$ be the $\varrho$ th element of $A_{\alpha}$. Then $f$ is a regressive function on $S_{\mathcal{A}}$ so by Fodor's theorem we have a stationary set $S \subset S_{\mathcal{A}}$ and a $\gamma \in \kappa$ with $f(\alpha)=\gamma$ for every $\alpha \in S$. But then, using $|\gamma|{ }^{\varrho}<\kappa$, we clearly have distinct $\alpha, \beta \in S$ with $\gamma \cap A_{\alpha}=\gamma \cap A_{\beta}$, hence $\left|A_{\alpha} \cap A_{\beta}\right| \geq \varrho$, contradicting $\mathcal{A}$ being $\varrho$-a.d.

REMARK. The above argument actually yields the following stronger result: Under the assumptions of 3.6 even $\uparrow(\kappa, \varrho \dot{+} \omega, \varrho)$ is false, with the obvious interpretation of this symbol. Thus we have arrived "down" to $\bullet(\kappa, \varrho, \varrho)$ that is "easy" to satisfy, being e.g. a consequence of the appropriate version of at $\kappa$ and $\varrho$. In fact, in many cases it holds even in ZFC.

We close this section with two simple results concerning the behaviour of $\boldsymbol{\uparrow}(\kappa, \lambda, \mu)$ in forcing extensions. The first one is a preservation result.

3.7. Proposition. Assume ${ }^{\bullet}(\kappa, \lambda, \mu)$ where $\kappa$ is regular and $P$ is a forcing notion with $|P|<\kappa$ such that both $\lambda$ and $\mu$ remain cardinals in $V^{P}$ ( $\kappa$ does so automatically). Then $\uparrow(\kappa, \lambda, \mu)$ remains valid in $V^{P}$.

Proof. Let $\mathcal{A}$ be a $\uparrow(\kappa, \lambda, \mu)$-family in $V$. Now $|P|<\kappa=\operatorname{cf}(\kappa)$ clearly implies that if $X \in[\kappa]^{\kappa}$ in $V^{P}$ then there is a $Y \in[X]^{\kappa} \cap V$, hence $A \subset Y \subset$ $X$ for some $A \in \mathcal{A}$, i.e. $\mathcal{A}$ remains a ${ }^{\bullet}(\kappa, \lambda, \mu)$-family in $V^{P}$.

The second result gives a method to obtain the consistency of $\uparrow\left(\chi, \varrho^{+}, \varrho\right)$ for a given regular cardinal $\varrho$, assuming that we have $\uparrow\left(\chi, \mu^{+}, \mu\right)$ for a singular cardinal $\mu$ of cofinality $\varrho$.

3.8. Proposition. Assume $\uparrow\left(\chi, \mu^{+}, \mu\right)$, where $\operatorname{cf}(\mu)=\varrho, \mu^{<\varrho}=\mu$, and $2^{\mu}<\chi=\operatorname{cf}(\chi)$. Then $\uparrow\left(\chi, \varrho^{+}, \varrho\right)$ holds in $V^{Q(\mu)}$.

Proof. Let $\mathcal{A} \subset[\chi]^{\mu^{+}}$be a ${ }^{\bullet}\left(\chi, \mu^{+}, \mu\right)$-family in the ground model $V$. Then, in $V^{Q(\mu)}$, applying 2.3 we have for every $A \in \mathcal{A}$ a subset $A^{*} \in[A] \varrho^{e^{+}}$ such that

$$
\mathcal{A}^{*}=\left\{A^{*}: A \in \mathcal{A}\right\}
$$

is $\varrho$-a.d. We claim that $\mathcal{A}^{*}$ is a $\uparrow\left(\chi, \varrho^{+}, \varrho\right)$-family. Since, by $2.2($ iii), we have $|Q(\mu)| \leq 2^{\mu}<\chi$, similarly to the proof of 3.7, every set $X \in[\chi]^{\chi}$ in $V^{Q(\mu)}$ has a ground model subset $Y$ with $|Y|=|X|=\chi$. But then there is an $A \in \mathcal{A}$ with

and the proof is complete.

$$
A^{*} \subset A \subset Y \subset X,
$$


4. The proof of 1.4. Assume GCH and that $\varrho=\operatorname{cf}(\varrho)<\kappa$, where $\kappa$ is 2 -huge. Concerning the property of 2 -hugeness we refer to 24.8 in [Ka], p. 332. In fact what we really need is the following property of $\kappa$ that is just a little more than being 1-huge:

There is an elementary embedding $j: V \rightarrow M$ with $\operatorname{crit}(j)=\kappa, j(\kappa)=\lambda$ and $M^{\lambda^{(+\varrho+3)}} \subset M$, or equivalently there is a $\kappa$-complete normal ultrafilter $\mathcal{D}^{*}$ over $\mathcal{P}\left(H\left(\lambda^{(+\varrho+3)}\right)\right)$ such that

$$
\left\{M: M \prec H\left(\lambda^{(+\varrho+3)}\right) \& M \cong H\left(\kappa^{(+\varrho+3)}\right)\right\} \in \mathcal{D}^{*} .
$$

We shall be working with the projection $\mathcal{D}$ of $\mathcal{D}^{*}$ to $H\left(\lambda^{(+\varrho+1)}\right)$, i.e.

$$
\mathcal{D}=\left\{A \subset H\left(\lambda^{(+\varrho+1)}\right):\left\{a \subset H\left(\lambda^{(+\varrho+3)}\right): a \cap H\left(\lambda^{(+\varrho+1)}\right) \in A\right\} \in \mathcal{D}^{*}\right\} .
$$

Then, of course, $\mathcal{D}$ is a $\kappa$ complete normal ultrafilter over $\mathcal{P}\left(H\left(\lambda^{+\varrho+1}\right)\right)$ such that

$$
X=\left\{M: M \prec H\left(\lambda^{(+\varrho+1)}\right) \& M \cong H\left(\kappa^{(+\varrho+1)}\right)\right\} \in \mathcal{D} .
$$

Write, for simplicity, $\mu=\kappa^{(+\varrho)}$ and $\chi=\lambda^{(+\varrho+1)}$. Combining the above with Solovay's result as in the final part of Section 2, we conclude that there is a stationary set $S \subset S_{\mu^{+}}^{\chi}$ such that for each $\delta \in S$ we have some $M_{\delta} \in X$ with $\bigcup\left(M_{\delta} \cap \chi\right)=\delta$, and moreover $\left\{M_{\delta}: \delta \in S\right\} \in \mathcal{D}$ is $\mu$-a.d. In what follows, we write $Y_{\delta}=M_{\delta} \cap \chi$ for $\delta \in S$.

The crucial part of our proof is the following result.

4.1. Lemma. There is a sequence $\left\langle f_{\delta}: \delta \in S\right\rangle$ such that

(i) $f_{\delta}: Y_{\delta} \rightarrow Y_{\delta}$ for each $\delta \in S$;

(ii) for every $f: \chi \rightarrow \chi$ the set $\left\{\delta \in S: f_{\delta} \subset f\right\}$ is stationary in $\chi$.

Proof. For $\delta \in S$, let $Y_{\delta}=\left\{\alpha_{\delta, \zeta}: \zeta \in \mu^{+}\right\}$be the increasing enumeration of $Y_{\delta}$. The functions $f_{\delta}: Y_{\delta} \rightarrow Y_{\delta}$ are defined by a simple transfinite recursion in such a way that for each $\delta \in S$ the set $H_{\delta}=\left\{\zeta \in \mu^{+}: f_{\alpha_{\delta, \zeta}} \uparrow\right.$ $\left.Y_{\delta} \cap Y_{\alpha_{\delta, \zeta}} \subset f_{\delta}\right\}$ be non-stationary in $\mu^{+}$, if this is possible at all.

All we have to do now is to check that (ii) holds. Assume, indirectly, that there exists $f: \chi \rightarrow \chi$ and $C \subset \chi$ c.u.b. such that $f_{\delta} \not \subset f$ for every $\delta \in S \cap C$.

For any $\alpha \in \chi$, as normality of $\mathcal{D}$ implies its fineness, we have

$$
A_{\alpha}=\left\{M_{\delta}: \alpha \in M_{\delta}\right\} \in \mathcal{D} .
$$

Also, for any pair $\langle\alpha, \beta\rangle \in \chi^{2}$ we can define $A_{\alpha, \beta} \in \mathcal{D}$ so that

$$
A_{\alpha, \beta}=\left\{\begin{array}{l}
\left\{M_{\delta}: f_{\delta}(\alpha)=\beta\right\} \\
\text { or } \\
\left\{M_{\delta}: f_{\delta}(\alpha) \neq \beta\right\} .
\end{array}\right.
$$


Then, by the normality of $\mathcal{D}$, there is a (clearly stationary) subset $S_{1} \subset S \cap C$ such that

$$
X_{1}=\left\{M_{\delta}: \delta \in S_{1}\right\} \in \mathcal{D}
$$

and if $\delta \in S_{1}, \alpha, \beta \in Y_{\delta}$ then $M_{\delta} \in A_{\alpha} \cap A_{\alpha, \beta}$.

Let $M_{\delta} \in X_{1} \cap A_{\alpha} \in \mathcal{D}$ where $\alpha \in \chi$. Then clearly $g(\alpha)=f_{\delta}(\alpha)$ does not depend on $\delta$, and moreover

$$
\left\{M_{\delta}: f_{\delta}(\alpha)=g(\alpha)\right\} \in \mathcal{D} .
$$

This implies that for every $\alpha \in \chi$ we have

$$
A_{\alpha, g(\alpha)}=\left\{M_{\delta}: f_{\delta}(\alpha)=g(\alpha)\right\}
$$

and consequently $f_{\delta} \subset g$ whenever $\delta \in S_{1}$. In particular, as $S_{1} \subset C$ and $f_{\delta} \not \subset f$ for $\delta \in C$, we have $f \neq g$.

Now, applying the normality of our original ultrafilter $\mathcal{D}^{*}$, we can find $N \prec H\left(\chi^{++}\right)$such that $N \cong H\left(\mu^{+++}\right)$, and moreover

(a) $\left\langle M_{\delta}: \delta \in S\right\rangle,\left\langle f_{\delta}: \delta \in S\right\rangle, S_{1}, f, g, C, \mathcal{D} \in N$;

(b) for any $Z \in N \cap \mathcal{D}$ we have $N \cap H(\chi) \in Z \cap X_{1}$;

(c) $N \cap H(\chi)=M_{\delta}^{*}$ with $\delta^{*} \in S_{1}$.

Let $h: N \rightarrow H\left(\mu^{+++}\right)$be the Mostowski collapse. Then $h(\chi)=\mu^{+}$. By elementarity $N \models$ " $S_{1}$ is stationary in $\chi$ ", hence $h\left(S_{1}\right)$ is stationary in $h(\chi)=\mu^{+}$, or in other words the set

$$
H=\left\{\zeta \in \mu^{+}: \alpha_{\delta^{*}, \zeta} \in S_{1}\right\}
$$

is stationary in $\mu^{+}$. But if $\alpha_{\delta^{*}, \zeta} \in S_{1}$ then we have $f_{\alpha_{\delta^{*}, \zeta}} \subset g$ as well as $f_{\delta^{*}} \subset g$, hence $f_{\alpha_{\delta^{*}, \zeta}} \backslash Y_{\delta^{*}} \cap Y_{\alpha_{\delta^{*}, \zeta}} \subset f_{\delta^{*}}$. So we conclude $H \subset H_{\delta^{*}}$, i.e. at step $\delta^{*}$ of the transfinite construction we could not make $H_{\delta^{*}}$ non-stationary.

However, as $f, S, C \in N$, on the other hand we have $f_{N}=f\left\lceil Y_{\delta^{*}}: Y_{\delta^{*}} \rightarrow\right.$ $Y_{\delta^{*}}$, and the set

$$
\left\{\zeta \in \mu^{+}: \alpha_{\delta^{*}, \zeta} \in C\right\}=h(C)
$$

is c.u.b. in $\mu^{+}$. By elementarity, for every $\zeta \in h(S \cap C)$ there is a $\gamma \in$ $N \cap M_{\alpha_{\delta^{*}, \zeta}}$ such that $f(\gamma) \neq f_{\alpha_{\delta^{*}, \zeta}}(\gamma)$, i.e.

$$
f_{\alpha_{\delta^{*}, \zeta}} \uparrow Y_{\delta^{*}} \cap Y_{\alpha_{\delta^{*}, \zeta}} \not \subset f_{N} \text {. }
$$

This, however, contradicts our above conclusion because $f_{N}$ would make, at step $\delta^{*}$, the set $H_{\delta^{*}}$ non-stationary in $\mu^{+}$.

Now from 4.1 we easily obtain the following result, where the notation is the same.

\subsection{Proposition. $\uparrow\left(\chi, \mu^{+}, \mu\right)$ is valid.}

Proof. Let $S^{*}=\left\{\delta \in S: f_{\delta}\right.$ is strictly increasing $\}$ and for each $\delta \in S^{*}$ let $Z_{\delta}=f_{\delta}{ }^{\prime \prime} Y_{\delta}$. We claim that $\mathcal{Z}=\left\{Z_{\delta}: \delta \in S^{*}\right\} \subset[\chi]^{\mu^{+}}$is a $\boldsymbol{\varphi}^{\prime}\left(\chi, \mu^{+}, \mu\right)$ family. Since $Z_{\delta} \subset M_{\delta}, \mathcal{Z}$ is clearly $\mu$-a.d. Now, for any set $Z \in[\chi]^{\chi}$ let $f$ be 
its increasing enumerating function. Then $S_{f}=\left\{\delta: f_{\delta} \subset f\right\}$ is stationary and also $S_{f} \subset S^{*}$. But for any $\delta \in S_{f}$ we clearly have $Z_{\delta} \subset Z$.

Now, it is very easy to complete the proof of 1.4. First note that 3.8 may be applied, i.e. in $V^{Q(\mu)}$ we have ${ }^{\bullet}\left(\chi, \varrho^{+}, \varrho\right)$. Next, as in $\S 2$, if one collapses $\lambda$ to $\varrho^{++}$in $V^{Q(\mu)}$ using $\operatorname{Lv}\left(\lambda, \varrho^{++}\right)$then the forcing $P=Q(\mu) * \operatorname{Lv}\left(\lambda, \varrho^{++}\right)$ is as required because it is $\varrho$-complete, preserves GCH, and $\bullet\left(\widehat{\varrho}, \varrho^{+}, \varrho\right)$ holds true in $V^{P}$. Indeed, the last part follows because $\chi=\widehat{\varrho}$ in $V^{P}$ and $\uparrow\left(\chi, \varrho^{+}, \varrho\right)$ is preserved by the Levy collapse (use 3.7 and $\left|\operatorname{Lv}\left(\lambda, \varrho^{++}\right)\right|<\chi$ ).

\section{References}

[BDJShSz] Z. T. Balogh, S. W. Davis, W. Just, S. Shelah and J. Szeptycki, Strongly almost disjoint sets and weakly uniform bases, Preprint no. 12 (1997/98), Hebrew Univ. Jerusalem, Inst. of Math.

[EH] P. Erdős and A. Hajnal, On a property of families of sets, Acta Math. Acad. Sci. Hungar. 12 (1961), 87-124.

[Gr] J. Gregory, Higher Souslin trees and the generalized continuum hypothesis, J. Symbolic Logic 41 (1976), 663-671.

[HJSh] A. Hajnal, I. Juhász and S. Shelah, Splitting strongly almost disjoint families, Trans. Amer. Math. Soc. 295 (1986), 369-387.

[Ka] A. Kanamori, The Higher Infinite, Springer, Berlin, 1994.

[Ko] P. Komjáth, Families close to disjoint ones, Acta Math. Hungar. 43 (1984), 199-207.

[S] R. Solovay, Strongly compact cardinals and the GCH, in: Proc. Sympos. Pure Math. 25, Amer. Math. Soc., 1974, 365-372.

[W] N. H. Williams, Combinatorial Set Theory, Stud. Logic 91, North-Holland, Amsterdam, 1977.

Department of Mathematics

Rutgers University

New Brunswick, NJ 08903, U.S.A.

E-mail: ahajnal@math.rutgers.edu

Mathematical Institute

of the Hungarian Academy of Sciences

P.O. Box 127

1364 Budapest, Hungary

E-mail: juhasz@math-inst.hu
Department of Mathematics Rutgers University New Brunswick, NJ 08903, U.S.A.

and

Institute of Mathematics

The Hebrew University

91904 Jerusalem, Israel E-mail: shelah@math.huji.ac.il

Received 7 December 1998;

in revised form 5 September 1999 